\title{
Prevention measures
}

\begin{abstract}
A proposed HIV prevention trial in African infants using the antibody therapy VRC01 offers hope, but some researchers say other antibodies might be more effective. Given the thorny nature of testing therapies in newborns and the limited funds for the rollout of HIV trials, advisory bodies such as the US Institute of Medicine would do well to weigh in on the ethical framework for pediatric trials of antibody drugs in resource-poor settings.
\end{abstract}

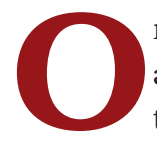

ne of the most dramatic examples of the need for pediatric medicines comes from the risk of mother-to-child transmission of HIV, a threat that lingers throughout breastfeeding. At the United Nations High Level Meeting on AIDS in New York in 2011, world leaders signed a pledge to eliminate mother-to-child transmission of the virus by 2015 . Yet there is a substantial way to go. That same year, an estimated 330,000 pediatric HIV infections occurred worldwide, most as a result of the virus passing from mother to child. And although antiretroviral drugs can lower virus counts in mothers and shield infants, it is thought that residual transmission of the virus might still occur.

To fully protect newborns in places of relatively high HIV pediatric incidence, new prevention modalities are needed. A proposed trial to test the broadly neutralizing antibody VRC01 aims to offer such a weapon-the first of its kind. The researchers behind the effort, including top scientists at the US National Institutes of Health (NIH), emphasize that the trial is not imminentthey are still in the process of preparing an Investigational New Drug Application to the US Food and Drug Administration for the phase 1 trial, which would assess the safety of a single dose in a group of adults in the US, and a smaller phase 1 study of US infants born to mothers infected with HIV. At present, the phase 2 trial is envisioned to include perhaps 3,000 newborns in countries such as Malawi, Tanzania and Uganda, and involve administering monthly doses of the antibody, combined with the antiretrovirals already given to this population, for at least the first six months of life.

The phase 1 trials would give researchers hard data on how the human body processes VRC01, which would, one hopes, minimize the risk of administering the antibody to newborns. And there is real-world evidence that monoclonal antibodies are tolerated and effective in a pediatric population: palivizumab, made by MedImmune, is a monoclonal given to prevent respiratory syncytial virus infection in vulnerable infants. Researchers hope that the antibodies will have a longer half-life than antiretroviral drugs and that VRC01 might provide added protection for infants who do not receive regular doses of antiretrovirals.
Despite general support for the VRC01 trial at a recent meeting in Uganda, not everyone is ready to back the idea of testing the antibody in infants. Monoclonals can trigger unexpected and grave adverse reactions-and the vulnerability of children cannot be overstated. Patient recruitment will be difficult, and recent experiments have hinted that antibodies might have potentially stronger neutralizing effects against HIV than VRC01.

There are also concerns about whether developing countries would be able to afford a monoclonal antibody should it come to market, as these therapies have traditionally had hefty prices. On one hand, the price of antiretrovirals has dropped precipitously in the past decade, thanks to scaled-up manufacturing and tiered pricing schemes, both of which could apply to VRC01 should it—or another monoclonal-prove effective. On the other hand, it is worth noting that the median sales price for the top 15 monoclonal antibodies and Fc fusion products was $\$ 8,000$ per gram in 2008 ( $m A$ Abs 1, 443-452, 2009). Given the difficulties of patient recruitment and the limited funds for HIV trials in resource-poor settings, it is neither feasible nor ethical to try each and every single promising antibody against HIV in infants. The question then becomes: who decides which antibody will go to trial? The race to sequence the human genome-in which the adoption of innovative techniques helped accelerate the process-illustrates that researchers at all levels need to engage in a continuous, open-minded discussion about which technology to take forward at each step. That lesson should be kept in mind for HIV-neutralizing antibodies. Millions of dollars have flowed toward the development of VRC01 by the NIH. It would help the research community, however, if an expert body, such as the US Institute of Medicine, were to convene a transparent ethical and scientific discussion about how to choose which antibody drugs to test in resource-poor settings-and then issue a report. The failure of an antibody trial in a pediatric population at risk for HIV infection could stifle the development of such therapies against this virus. With so much at stake, formal ethical guidance that offers criteria for deciding which antibodies to pursue may help neutralize worries. 